\title{
Congestive Heart Failure Patients' Pulse Rate Progression and Time to Death at Debre Tabor Referral Hospital, Ethiopia
}

\author{
Alebachew Taye Belay ${ }^{D},{ }^{1}$ Denekew Bitew Belay, ${ }^{2}$ Shewayiref Geremew Gebremichael, ${ }^{3}$ \\ and Setegn Bayabil Agegn ${ }^{1}$ \\ ${ }^{1}$ Department of Statistics, Debre Tabor University, Debre Tabor, Ethiopia \\ ${ }^{2}$ Department of Statistics, Bahir Dar University, Bahir Dar, Ethiopia \\ ${ }^{3}$ Ethiopian Public Health Institute, Addis Ababa, Ethiopia
}

Correspondence should be addressed to Alebachew Taye Belay; alex016stat@gmail.com

Received 29 June 2021; Accepted 25 October 2021; Published 30 November 2021

Academic Editor: Carol J. Burns

Copyright (C) 2021 Alebachew Taye Belay et al. This is an open access article distributed under the Creative Commons Attribution License, which permits unrestricted use, distribution, and reproduction in any medium, provided the original work is properly cited.

\begin{abstract}
Background. Heart failure is a progressive condition marked by worsening symptoms such as shortness of breath, coughing, exhaustion and lethargy, fluid retention with swelling of the legs and abdomen, and a reduced ability to exercise. As a result, this study aims to use a joint model application to determine the joint risk factors of longitudinal change in pulse rate and time to death of congestive heart failure patients and their association admitted to a hospital. Methods. A retrospective study was undertaken on congestive heart failure patients admitted to the Debre Tabor Referral Hospital from January 2016 to December 2019. A statistical joint modeling strategy was employed to match the repeated biomarker pulse rate and a survival outcome at the same time. A total of 271 patients with congestive heart failure were chosen. Data were analyzed with $R$ statistical software via joineRML. Results. According to the findings, the association between longitudinal changes in pulse rate and time to death in heart failure patients is statistically significant. Sex, residence, left ventricular injection fraction, New York Heart Association class, and diabetes mellitus were all found to be significant risk factors for congestive heart failure patients' short survival time to death. Age, sex, residence, hypertension, left ventricular injection fraction, congestive heart failure, diabetes mellitus, tuberculosis, and etiology were all significant contributors in pulse rate progression. Conclusion. The computed association parameters revealed subject-specific values. The subject-specific linear time slope of PR measurement was positively related to the hazard rate of time to death of CHF patients in the study area. To reduce the risk level of CHF, health professionals, governmental organizations, and nongovernmental organizations must promote and allocate a suitable amount of budget for the treatment of CHF patients.
\end{abstract}

\section{Introduction}

Heart failure (HF) is a potentially fatal clinical consequence characterized by structural or functional dysfunction of the pericardium, myocardium, heart valves, and metabolic imbalances in the body $[1,2]$. Shortness of breath, coughing, exhaustion and lethargy, fluid retention with swelling of the legs and abdomen, and diminished physical exercise ability are all symptoms of heart failure [3]. HF is a leading cause of premature morbidity and mortality, with a lifetime risk of $20-46 \%$ [4], and it continues to be a global epidemic affecting millions of people $[5,6]$. It affects an estimated 33 million people around the world or $26.4 \%$ of the adult population, with $65.73 \%$ living in industrialized countries and $34.27 \%$ in developing nations [7].

According to a study conducted in 2014, the global prevalence of heart failure is estimated to be around 26 million patients [8]. Because this does not account for undetected cases of heart failure, a true estimate of the global burden of heart failure is unknown, although it is considered to be substantial [8]. Mortality rates for heart failure patients remain high, with 17 to $45 \%$ of deaths happening within one year of diagnosis and the majority of fatalities occurring within five years [3]. The prevalence of CHF in the adult 
population in the developed world is approximately 1 to $2 \%$, and it rises to more than $10 \%$ in those aged 70 years or older [9].

Heart failure is a well-recognized public health problem in industrialized countries such as the United States and Europe, reflecting a substantial burden of disease, and healthcare systems are the top causes of hospitalization and death in countries $[10,11]$. Other studies revealed that more than 5.8 million people in the United States had CHF $[10,12]$. Furthermore, heart failure afflicted over 6 million adults in the United States [13], and according to the 2013 death rate report, more than 2200 individuals died per day owing to cardiovascular illness, with heart failure being the underlying reason for the bulk of those deaths [14]. Heart failure is a particularly difficult ailment in Sub-Saharan Africa, and it occurs at a younger age than in other regions of the world. When access to echocardiography or serological markers is limited, misdiagnosis of HF is more likely [15]. Treatment trends indicate that evidence-based treatments are underutilized, compliance is low, and the hospital case fatality rate among people with HF in Sub-Saharan Africa ranges from 9 to $12.5 \%$ [15].

The prevalence of risk factors linked with heart failure is increasing in low-income areas like Sub-Saharan Africa (SSA), and it has emerged as the most prevalent primary diagnosis for patients referred to hospitals in Africa with suspected cardiac disease [16]. According to a recent study, patients with heart failure in Africa were the youngest and most likely to be in New York Health Association functional class IV when compared to those in Asia, the Middle East, and South America [17]. The Sub-Saharan Africa Survey of Heart Failure [18] study identified a growing cause of heart failure from 23 to $43 \%$ in persons with heart failure. Diagnosis of heart failure might pose substantial issues in Africa due to a lack of access to proper diagnostics. With the rising trend in HF-related morbidity and death across Africa, especially Ethiopia $[16,19]$, there is a paucity of data on heart failure patients' self-care performance. According to the WHO [20], CHF was responsible for around 9\% of all fatalities in Ethiopia in 2012. Depending on the diagnostic criteria employed and the population investigated, the global incidence of HF ranges from 100 to 900 cases per 100,000 person-years [21].

Monitoring the burden of congestive heart failure is required for patients to jointly assess the patient's health and survival time. To investigate the relationship between the two outcomes, the joint model was employed to assess the longitudinal biomarker and survival outcomes concurrently [22-24]. When observations over time are correlated, it enhances statistical inference efficiency and decreases bias [25]. Because of the time-to-event outcomes associated with longitudinal trajectories throughout time, separate analyses of longitudinal biomarkers and survival events may result in inefficient or biased conclusions. Joint models of longitudinal biomarkers and survival events incorporate all information at the same time, resulting in reliable and efficient inferences [26]. Joint model interest frequently rests in understanding the relationship between a process's longitudinal history and its effect on the risk of an event
[24, 27-31], and it enables more powerful, accurate, efficient, and robust estimations [26, 28, 32, 33]. Monitoring factors and the burden of congestive heart failure is essential to determine the patient's health and survival time.

As a result, the goals of this study are to determine the joint risk factors of longitudinal change in pulse rate and time to death in congestive heart failure patients admitted to Debre Tabor Referral Hospital in Ethiopia, as well as their association, using a joint model application.

\section{Data and Methods}

2.1. Study Area. This study was carried out at the Debre Tabor Referral Hospital (DTRH), which is located in NorthWest Ethiopia. This hospital serves as a referral hospital for the people in the surrounding area.

2.2. Study Design. To achieve the study's aims, a retrospective study was done to gather essential information from the medical files of patients with HF.

2.3. Data Source. The population for this trial was made up of DTRH patients with congestive heart failure. Data were acquired from the medical charts of DTRH congestive heart failure patients from January 1, 2016, to December 31, 2019. The timing of the death of congestive heart failure patients was the survival endpoint of interest.

2.4. Study Variable. The longitudinal changes in pulse rate and the survival time of $\mathrm{CHF}$ patients after initiating medication were the study's response variables.

Gender, residence, weight, type of $\mathrm{CHF}$ in patients, etiology of HF, NYHA classification, LVEF, observation time, presence of diabetes mellitus disease, presence of hypertension disease, presence of pneumonia disease, presence of tuberculosis disease, and presence of chronic kidney disease were all factors considered (see Table 1).

2.5. Statistical Analysis. The joint model of the longitudinal linear mixed-effect model and the event time model was investigated concurrently in this study. A statistical choice was taken at a 5\% level of significance, and the analysis was carried out using $R$ version 4.0.0. A linear mixed-effect (LME) model for longitudinal data and a Cox proportional hazard $(\mathrm{PH})$ model for time-to-event data, with an association parameter, shows the effect of true longitudinal measurement on CHF time to death [26]. This is often referred to as the shared parameter $\alpha$ model approach.

\section{Results}

3.1. Data Exploratory. The number of patients reduced from 271 at the start to 11 at the end of the 16 consecutive visiting occasions. HF patients had mean and median survival periods of 18.83 and 18 months, respectively. From a total of 271 CHF patients, 56 (20.7\%) died, while 216 (79.3\%) were censored (Table 1). 
TABLE 1: Baseline categorical variable characteristics for CHF patients with DTRH from January 1, 2016, to December 31, 2019.

\begin{tabular}{|c|c|c|c|c|c|}
\hline No. & Variable & Categories & Censored (\%) & Observed event (\%) & Total (\%) \\
\hline \multirow{2}{*}{1} & \multirow{2}{*}{ Sex } & Male & $66(24.4)$ & $34(12.5)$ & $100(36.9)$ \\
\hline & & Female & $149(55.0)$ & $22(8.1)$ & $171(63.1)$ \\
\hline \multirow{3}{*}{2} & \multirow{2}{*}{ Residence } & Rural & $64(23.6)$ & $21(7.7)$ & $85(31.4)$ \\
\hline & & Urban & $151(55.7)$ & 35 (12.9) & $186(68.6)$ \\
\hline & & Left ventricular & $61(22.5)$ & $36(13.3)$ & $97(35.8)$ \\
\hline \multirow[t]{3}{*}{3} & Type of CHF patients & Right ventricular & $81(29.9)$ & $13(4.8)$ & $94(34.7)$ \\
\hline & & Biventricular & $73(26.9)$ & $7(2.6)$ & $80(29.5)$ \\
\hline & & Class II & $63(23.2$ & $2(0.7)$ & $65(24.0$ \\
\hline \multirow[t]{2}{*}{4} & New York Heart Association class of CHF patients & Class III & $78(28.8)$ & $20(7.4)$ & $98(36.2)$ \\
\hline & & Class IV & $74(27.3)$ & $34(12.4)$ & $108(39.9)$ \\
\hline \multirow{2}{*}{5} & \multirow{2}{*}{ Presence of hypertension } & Yes & $47(17.3)$ & $7(2.6)$ & $54(19.9)$ \\
\hline & & No & $168(62.0)$ & $49(18.1)$ & $217(80.1)$ \\
\hline \multirow{2}{*}{6} & \multirow{2}{*}{ Presence of pneumonia } & Yes & $32(11.9)$ & $8(3.0)$ & $40(14.8)$ \\
\hline & & No & $182(67.4)$ & $48(17.8)$ & $230(85.2)$ \\
\hline \multirow{2}{*}{7} & \multirow{2}{*}{ Presence of diabetes mellitus } & Yes & $40(14.8)$ & $29(10.7)$ & $69(25.5)$ \\
\hline & & No & $175(64.6)$ & $27(10.0)$ & $202(74.5)$ \\
\hline \multirow{2}{*}{8} & \multirow{2}{*}{ Presence of chronic kidney disease } & Yes & $22(8.1)$ & $6(2.2)$ & $28(10.3)$ \\
\hline & & No & $193(71.2)$ & $50(18.5)$ & $243(89.7)$ \\
\hline \multirow{4}{*}{9} & \multirow{2}{*}{ Presence of tuberculosis } & Yes & $42(15.5)$ & $41(15.1)$ & $83(30.6)$ \\
\hline & & No & $173(63.8)$ & $15(5.5)$ & $188(69.4)$ \\
\hline & & VHD & $70(25.8)$ & $20(7.4)$ & $90(33.2)$ \\
\hline & & HHD & $44(16.2)$ & $9(3.3)$ & $53(19.6)$ \\
\hline \multirow[t]{3}{*}{10} & Etiology of heart failure & IHD & $27(10.0)$ & $5(1.8)$ & $32(11.8)$ \\
\hline & & Dilated cardiomyopathy & $51(18.8)$ & $13(4.8)$ & $64(23.6)$ \\
\hline & & Other etiologies & $23(8.5)$ & $9(3.3)$ & $32(11.8)$ \\
\hline \multirow{2}{*}{12} & & Censored & $215(79.3)$ & $271(100.0 \%)$ & \\
\hline & Status of survival time or the study & Event (death) & $56(20.7)$ & & \\
\hline
\end{tabular}

$\mathrm{VHD}=$ valvular heart disease, $\mathrm{HHD}=$ hypertensive heart disease, and $\mathrm{IHD}=$ ischemic heart disease.

The average age of CHF patients admitted to the hospital was 50 years, with a standard deviation of 18.53 years, while the patients' minimum and maximum ages were 18 and 86 years, respectively. The mean weight of $\mathrm{CHF}$ patients was $58.10 \mathrm{~kg}$, with a standard deviation of $11.24 \mathrm{~kg}$, and the lowest and highest weights were 35.0 and $93.0 \mathrm{~kg}$, respectively. The mean LVEF of CHF patients was 51.29 with a standard deviation of 16.32, while the minimum and maximum LVEF were 13 and 84, respectively (Table 2).

3.2. Data Exploration. The longitudinal PR measure appears to satisfy the assumption of normality, and the box plot for the longitudinal PR shows that the PR's normality assumptions were approximately normally distributed, as indicated by the relative position of the median within the box that contained half of the data that satisfied the assumption of normality (Figures 1 and 2).

The profile plot of HF patients shows the diversity in PR over time within and across patients. Because the measurements were not evenly distributed amongst the subjects, the data were not balanced. The profile plot also shows that the horizontal loss smoothing approach indicates that the mean structure of PR is roughly linear over time (Figures 3 and 4).

3.3. Model Comparison. The AIC and BIC values in the firstorder autoregressive (AR (1)) covariance structure are lower than those in the other covariance structures in PR. This implies that the AR (1) covariance structure is the best fit for our data when compared to the other covariance structures for the longitudinal change in PR of CHF data (Table 3).

Based on its lower AIC (15528.45) and BIC values, the linear mixed-effect model with longitudinal changes in PR considered the model with random intercept and linear temporal slope to be the most parsimonious model for the linear mixed-effect model (15623.31) (Table 4).

3.4. Joint Modeling Approach. In the univariable analysis of pulse rate $(\mathrm{PR})$, the main effects of sex, age, residence, observation period, LVEF, NYHA class of patients, type of CHF, DM, TB, etiology, and hypertension were significant at a level of significance of 0.25 . Gender, residence, NYHA class, baseline weight, CHF type, LVEF, presence of HTN, $\mathrm{CKD}, \mathrm{TB}$, and $\mathrm{DM}$ were found as significant predictors by the log-rank and univariable Cox models at 25\% for creating a multivariable Cox model. The statistical joint model analysis of pulse rate and survival time to death was performed using $R$ software and the joineRML package, and the results are provided in Table 5.

The length of follow-up time for CHF patients had a statistically significant effect on the change in PR over time. When all other model parameters were kept constant, the average longitudinal change in $\mathrm{PR}$ in $\mathrm{CHF}$ patients was 0.677 bpm $(p$ value $=0.002)$. A statistically significant longitudinal change in PR was connected with the age of CHF patients. The age of CHF patients was statistically significant in a 
TABLE 2: Baseline characteristics of continuous variables of HF patients.

\begin{tabular}{|c|c|c|c|c|c|}
\hline Variables & $\mathrm{N}$ & Minimum & Maximum & Mean & Stdev \\
\hline Baseline age (year) & 271 & 18 & 86 & 50.00 & 18.53 \\
\hline Baseline weight $(\mathrm{kg})$ & 271 & 35 & 93 & 58.10 & 11.24 \\
\hline Left ventricle ejection fraction (\%) & 271 & 13 & 84 & 51.29 & 16.32 \\
\hline
\end{tabular}

Stdev: standard deviation.

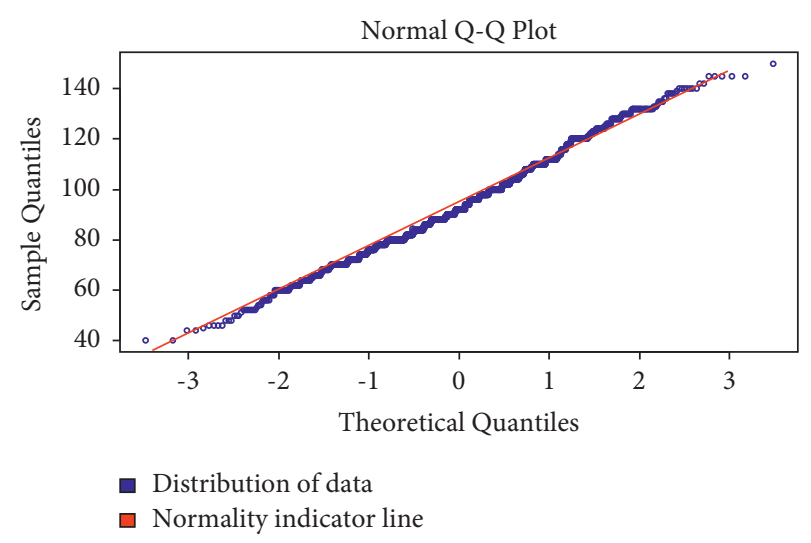

Figure 1: Normal Q-Q plot of the pulse rate for heart failure patients.

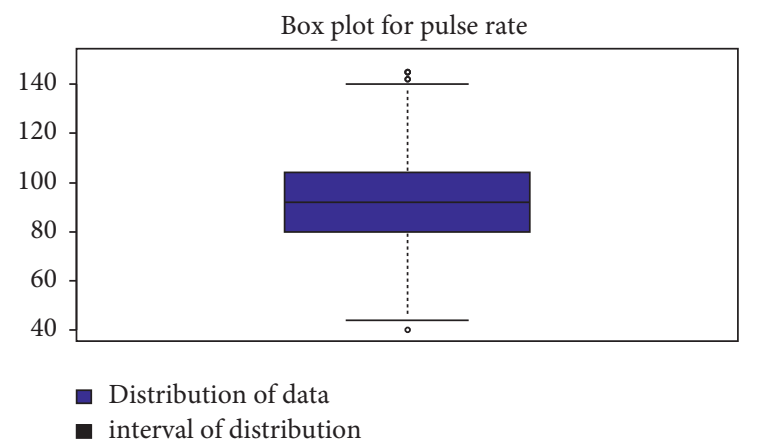

Figure 2: Box plot of the pulse rate for heart failure patients.

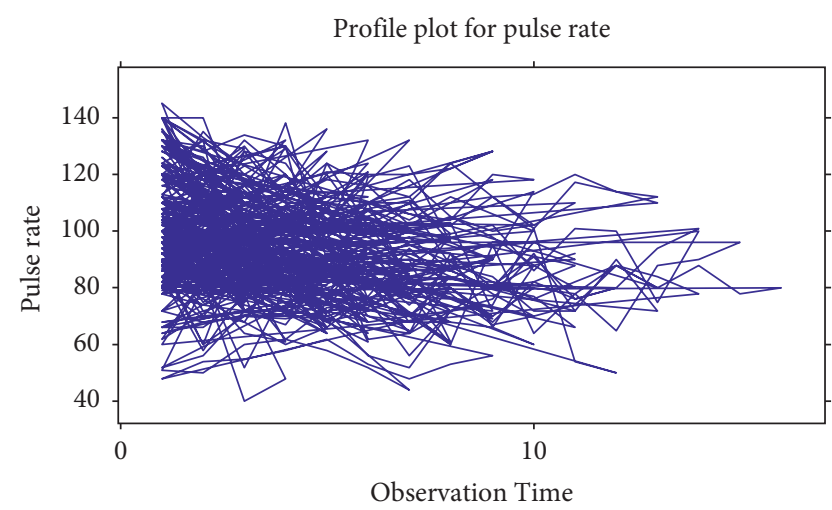

FIgURe 3: Profile plot of the pulse rate for heart failure patients.

mixed-effect model of the longitudinal pulse rate change. When all other risk factors were kept constant, the average longitudinal change in PR of CHF patients decreased by

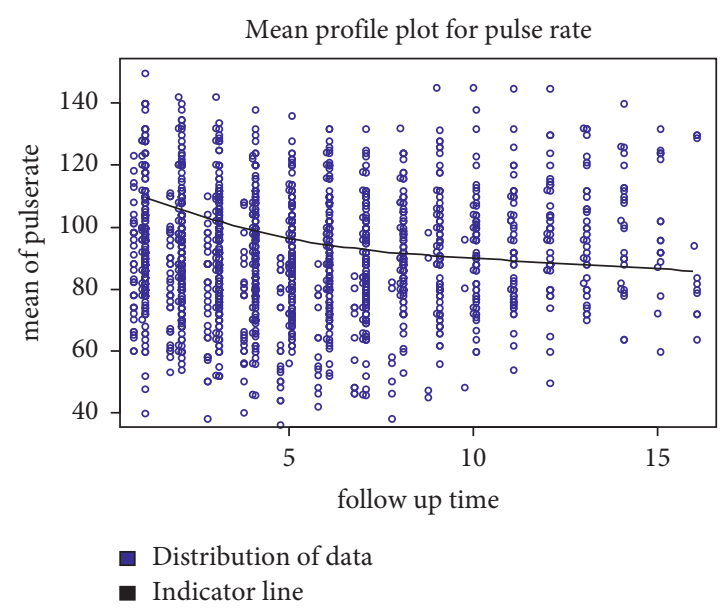

Figure 4: Mean profile plot of the pulse rate for heart failure patients.

TABLE 3: Comparison of the covariance structure of a linear mixedeffect model.

\begin{tabular}{lccc}
\hline Covariance structure & \multicolumn{3}{c}{ Pulse rate } \\
& AIC & BIC & LogLik \\
\hline CS & 15627.27 & 15722.14 & -7796.64 \\
AR $(1)$ & 15528.45 & 15623.31 & -7747.22 \\
UN & 15625.27 & 15714.56 & -7796.64 \\
TOEP & 15591.75 & 15681.13 & -7779.87 \\
\hline
\end{tabular}

$0.097 \mathrm{bpm}(p$ value $=0.021)$ as their baseline age increased by a year (Table 5).

Gender has a significant relationship with the average longitudinal change of pulse rate, and male patients increased the average longitudinal change of PR by $5.91 \mathrm{bpm}$ ( $p$ value $=0.003$ ) when all other parameters in the model were kept constant.

The average longitudinal change of PR is very different depending on where you live. When all other parameters were kept equal, urban patients had a $3.59 \mathrm{bpm}$ lower average longitudinal change in PR ( $p$ value $=0.006)$ than rural patients.

The baseline LVEF of CHF patients increased by $1 \%$, but the average longitudinal change in PR dropped by $16.1 \%$ bpm $(p$ value $<0.001)$, while all other variables remained constant. The left ventricular type of the congestive heart failure patient significantly reduced the average longitudinal change in PR by $3.871 \mathrm{bpm}(p$ value $=0.012)$ compared to biventricular type in CHF patients, while all other parameters were similar. Being a NYHA class IV CHF patient increased the average longitudinal change in PR by 8.314 
TABLE 4: Selection of random effects to be included in the LMM for PR and RR CHF patients' data.

\begin{tabular}{lccc}
\hline Model for random effect & AIC & BIC & LogLik \\
\hline Random intercept only model & 15627.27 & 15722.14 & -7796.64 \\
Random slope only model & 15528.45 & 15623.31 & -7747.22 \\
Random intercept and random slope model & 15625.27 & 15714.56 & -7796.64 \\
\hline
\end{tabular}

TABLE 5: Joint model analysis of PR and the time to death of CHF patient's data at DTRH.

\begin{tabular}{|c|c|c|c|c|c|c|c|c|}
\hline \multicolumn{4}{|c|}{ Longitudinal process } & \multicolumn{5}{|c|}{ Survival process } \\
\hline Parameter & $\widehat{\beta}$ & Se $(\widehat{\beta})$ & $p$ value & Parameter & $\widehat{\beta}$ & $\operatorname{Se}(\widehat{\beta})$ & HR & $p$ value \\
\hline Intercept & 103.15 & 4.71 & $<0.001$ & Sex (male) & 1.393 & 0.761 & 4.027 & 0.028 \\
\hline Age & -0.097 & 0.042 & 0.021 & Residence (urban) & 0.090 & 0.842 & 1.094 & 0.020 \\
\hline Residence (urban) & -3.590 & 1.870 & 0.006 & LVEF & -0.026 & 0.011 & 0.974 & 0.027 \\
\hline Sex (male) & 5.910 & 1.960 & 0.003 & Weight & -0.017 & 0.017 & 0.983 & 0.326 \\
\hline HTN (yes) & 1.517 & 1.760 & 0.039 & HTN (yes) & 0.737 & 0.920 & 2.090 & 0.423 \\
\hline \multicolumn{9}{|c|}{ CHF type (ref = biventricular) } \\
\hline Right ventricular & -2.679 & 2.130 & 0.209 & Right ventricular & -0.573 & 0.823 & 0.564 & 0.485 \\
\hline Left ventricular & -3.871 & 2.057 & 0.012 & Left ventricular & 0.614 & 0.573 & 1.848 & 0.284 \\
\hline LVEF & -0.161 & 0.047 & $<0.001$ & NYHA class & & & & \\
\hline NYHA (ref = class II) & & & & Class III & 3.010 & 2.957 & 20.290 & $<0.001$ \\
\hline Class III & 3.632 & 2.239 & 0.105 & Class IV & 3.570 & 2.773 & 35.520 & $<0.001$ \\
\hline Class IV & 8.314 & 2.159 & $<0.001$ & CKD (yes) & -0.600 & 0.946 & 0.549 & 0.526 \\
\hline DM (yes) & 5.314 & 2.011 & 0.008 & DM (yes) & 0.313 & 0.439 & 1.368 & 0.005 \\
\hline $\mathrm{TB}$ (yes) & 7.017 & 2.503 & 0.005 & TB (yes) & 1.141 & 0.631 & 3.130 & 0.071 \\
\hline Etiology $($ ref = VHD $)$ & & & & Gamma_1 $(\alpha)$ & 0.018 & 0.009 & 1.018 & 0.045 \\
\hline HHD & -6.399 & 2.424 & 0.008 & & & & & \\
\hline IHD & 0.991 & 2.845 & 0.728 & & & & & \\
\hline Cor pulmonale & -2.061 & 3.058 & 0.500 & & & & & \\
\hline Dilated cardiomyopathy & -2.439 & 2.561 & 0.341 & & & & & \\
\hline Other etiologies & -1.928 & 2.751 & 0.484 & & & & & \\
\hline Observation time & -0.677 & 0.216 & 0.002 & & & & & \\
\hline Random effect & Std. dev & & & & & & & \\
\hline Intercept $\left(\mathrm{b}_{0 \mathrm{i}}\right)$ & 202.930 & & & & & & & \\
\hline Obstime $\left(\mathrm{b}_{1 \mathrm{i}}\right)$ & 5.795 & & & & & & & \\
\hline $\operatorname{Cov}\left(b_{0}, b_{1 i}^{11}\right)^{11}$ & -26.890 & & & & & & & \\
\hline Residual $\left(\varepsilon_{\mathrm{i}}\right)$ & 9.914 & & & & & & & \\
\hline
\end{tabular}

$\mathrm{CHF}=$ congestive heart failure, $\mathrm{CKD}=$ chronic kidney disease, $\mathrm{DM}=$ diabetes mellitus, $\mathrm{HHD}=$ hypertensive heart disease, HTN $=$ hypertension, $\mathrm{IHD}=$ ischemic heart disease, $\mathrm{LVEF}=$ left ventricular ejection fraction, $\mathrm{NYHA}=$ New York Heart Association, $\mathrm{PR}=$ pulse rate, Ref $=$ reference category, $\mathrm{Se}=$ standard error, $\mathrm{TB}=$ tuberculosis, and $\mathrm{VHD}=$ valvular heart disease.

bpm ( $p$ value $<0.001)$ compared to being a NYHA class II patient when other factors kept constant.

When all other parameters were maintained constant, patients with congestive heart failure who had DM illness showed increase in their average longitudinal change in PR by $5.314 \mathrm{bpm}$ ( $p$ value $=0.008$ ) compared to patients who did not have DM illness. When all other parameters were maintained constant, patients with congestive heart failure who had TB disease showed increase in their average longitudinal change in PR by $7.017 \mathrm{bpm}$ ( $p$-value $=0.005)$ compared to patients who did not have TB disease. When all other parameters were maintained constant, patients with congestive heart failure who had HTN disease showed increase in their average longitudinal change in PR by $1.517 \mathrm{bpm}(p$ value $=0.039)$ compared to patients who did not have HTN illness. When all other parameters were kept constant, HHD as the etiology of CHF patients lowered the average longitudinal change in $\mathrm{PR}$ by $6.399 \mathrm{bpm}$ ( $p$ value $=0.008)$ compared to VHD as the etiology of CHF patients.
The variation of the random intercepts in $P R$ for the random portion of the linear mixed-effect model was 202.93, with a random slope of 5.795. This indicates that there is a greater baseline difference in PR at the start of their treatment. With the random intercept covariance, the random slope of the biomarker PR was -26.89 and the variance of the joint model was 9.914 (Table 5).

In the combined model, the estimated value of the association parameter alpha was $0.018(\mathrm{HR}=1.018, p=0.045)$, indicating that the average longitudinal change in PR had a positive relationship with the time to death of $\mathrm{CHF}$ patients across time.

Being male was statistically related to an increased risk of death in CHF patients $(\mathrm{HR}=4.027$, $p$ value $=0.0277)$, implying that male patients had 4.027 times higher risk of death than female patients when all other factors in the model were kept constant. Being an urban patient was statistically related to an increased risk of mortality in CHF patients $(\mathrm{HR}=1.094, p$ value $=0.020)$, which means that urban 
patients had 1.094 times higher risk of death than rural patients when all other factors in the model were kept constant.

\section{Discussion}

The average longitudinal change in PR demonstrated a negative relationship with the length of follow-up for $\mathrm{CHF}$ patients. This conclusion is also consistent with the earlier finding obtained by [34]. The average longitudinal change in PR had a statistically significant detrimental influence on the age of CHF patients. This study's findings contrast those of a previous study conducted in [34]. Age shows a substantial positive correlation with the prevalence of HF [35]. According to another study [36], younger people may have less $\mathrm{HF}$ understanding and become at risk.

The left ventricular ejection fraction had a negative significant influence on the average longitudinal change in $\mathrm{PR}$ and was linked to a higher risk of death in CHF patients. This finding was consistent with previous research [34]. However, a study conducted in [36] found no indication of a link between LVEF and quality of life in HF patients. Another study [37] revealed that LVEF illness did not affect HF mortality.

The rise in the New York Heart Association class of CHF patients had a positive significant influence on the average PR evaluation of CHF patients admitted to the hospital and was related to an increased risk of death in $\mathrm{CHF}$ patients. This outcome was consistent with the findings of [34]; other studies [36] found that greater NYHA class worsened the quality of life of HF patients. Another study [38] showed that NYHA has no significant effect on the mortality of HF.

Being male had a substantial favorable effect on the average longitudinal change of PR and was a risk factor for death in CHF patients. This finding is consistent with the prior finding in [36], which found that males had a positive correlation with lower quality of life. However this result contradicts a previous study [34] and other research [35] that found a favorable correlation between females and the prevalence of HF. Another study conducted in [39] discovered that gender has no significant effect on HF mortality.

When compared to rural settings, patients who lived in an urban area had a negative significant influence on the average longitudinal change in PR and risk factors for death. This study supported [36], which found that the quality of life in rural heart failure patients was lower than in urban patients due to poorer perceived control and symptoms of the disease. Patients with congestive heart failure who had DM disease had a beneficial influence on the average longitudinal change of $\mathrm{PR}$ and were at a higher risk of death. This result supports prior research [40] that has shown that the presence of DM has a positive and significant effect on the quality of HF. However, other studies [38] found that DM has no significant effect on HF.

Patients with congestive heart failure who had HTN disease showed a positive significant influence on the average longitudinal change in PR. This result supports prior research $[35,39,41,42]$ that found HTN to be highly linked with heart failure and to have a favorable significant influence on heart failure. Having an etiology of HHD was connected with a lower PR of congestive heart failure. This is inconsistent with a prior study [41] that found no significant relationship between $\mathrm{HF}$ and any of the etiologies of HF (ischemic heart disease, dilated cardiomyopathy, hypertensive heart disease, valvular heart disease, and other etiologies) except cor pulmonale.

\section{Conclusion}

The linear mixed-effect model with the subject value of PR measurement (in bpm) was a good fit for the longitudinal change PR evaluation in this investigation. At a 5\% level of significance, the cofactors including baseline age, gender, residency, CHF type, NYHA class, DM, HTN, and TB illness, LVEF, etiology, and observation period were statistically significant factors that affect the mean longitudinal change of PR measures of the patients. The Cox PH model showed a good fit for the time to death of patients.

The fitted Cox model results revealed that sex, residency, LVEF, NYHA class, and DM disease were the factors that influenced the hazard rate of time to death of CHF patients in the study area at a $5 \%$ level of significance. However, the independent variables such as weight, TB, CKD, and $\mathrm{CHF}$ type have no association with the hazard rate of time to death of $\mathrm{CHF}$ patients.

For the joint modeling of the data, the joint model with random intercepts and time of slope from the longitudinal model and the survival model was appropriate. The computed association parameters revealed subject-specific values, and the subject-specific linear time slope of PR measurement was found to be positively related to the hazard rate of time to death of CHF patients in the study area. To reduce the risk level of $\mathrm{CHF}$, health professionals, governmental organizations, and nongovernmental organizations must promote and allocate a suitable amount of budget for the treatment of CHF patients.

\section{Abbreviations}

AIC: Akaike information criterion

AR: Autoregressive order

BIC: Bayesian information criteria

CHF: Congestive heart failure

CKD: Chronic kidney disease

CS: Compound symmetry

DM: Diabetes mellitus

DTRH: Debre Tabor Referral Hospital

HHD: Hypertensive heart disease

HTN: Hypertension

IHD: Ischemic heart disease

LME: Linear mixed effect

LVEF: Left ventricular ejection fraction

NYHA: New York Heart Association

PR: $\quad$ Pulse rate

REML: Restricted maximum likelihood

TB: Tuberculosis

SD: Standard deviation 
UN: Unstructured

VHD: Valvular heart disease

WHO: World Health Organization.

\section{Data Availability}

The data, which are now in the hands of the original author, will be made available to public upon request.

\section{Ethical Approval}

Ethical approval was acquired from the ethical review board of Bahir Dar University's College of Sciences, and authorization for data collection was acquired from the Debre Tabor Referral Hospital Patient's Ethical Committee.

\section{Consent}

Each patient additionally provided informed consent before data collection.

\section{Conflicts of Interest}

The authors declare no conflicts of interest.

\section{Authors' Contributions}

AT was involved in the study design, performed the data extraction, analyzed the data, and produced the manuscript; DB was involved in the study design, counseled at each stage, and read the paper. AT, DB, and SG contributed to the manuscript's development. The final paper was critically evaluated and approved by all authors.

\section{Acknowledgments}

The authors would like to thank Debre Tabor Referral Hospital employees for supplying vital information and unwavering assistance, as well as data collectors.

\section{References}

[1] C. W. Yancy, M. Jessup, B. Bozkurt et al., "2016 ACC/AHA/ HFSA focused update on New pharmacological therapy for heart failure: an update of the 2013 ACCF/AHA guideline for the management of heart failure," Journal of the American College of Cardiology, vol. 68, no. 13, pp. 1476-1488, 2016.

[2] C. W. Yancy, "ACCF/AHA guideline for the management of heart failure: a report of the American College of cardiology foundation/American heart association task force on practice guidelines," Journal of the American College of Cardiology, vol. 62, no. 16, pp. e147-e239, 2013.

[3] P. Ponikowski, A. A. Voors, S. D. Anker et al., "2016 ESC Guidelines for the diagnosis and treatment of acute and chronic heart failure," European Heart Journal, vol. 37, no. 27, pp. 2129-2200, 2016.

[4] M. D. Huffman, "The Lifetime risk for heart failure among white and black Americans cardiovascular lifetime risk pooling project," Journal of the American College of Cardiology, vol. 61, no. 14, pp. 1510-1517, 2013.
[5] A. L. Bui, T. B. Horwich, and G. C. Fonarow, "Epidemiology and risk profile of heart failure," Journal Nature Reviews Cardiology, vol. 8, no. 1, p. 30, 2011.

[6] J. E. van de Mortel, R. C. H. de vos, E. Dekkers et al., "Metabolic and transcriptomic changes induced in Arabidopsis by the rhizobacterium Pseudomonas fluorescens SS101," Plant Physiology (Bethesda), vol. 160, no. 4, pp. 2173-2188, 2012.

[7] H. T. Peters, J. Pisegna, J. Faieta, and S. J. Page, "Functional brain stimulation in a chronic stroke survivor with moderate impairment," The American Journal of Occupational Therapy, vol. 71, no. 3, pp. 7103190080p1-7103190080p6, 2017.

[8] A. P. Ambrosy, G. C. Fonarow, J. Butler et al., "The global health and economic burden of hospitalizations for heart failure," Journal of the American College of Cardiology, vol. 63, no. 12, pp. 1123-1133, 2014.

[9] S. Claridge, A. Chakrabarti, K. Greaves, and C. J. Boos, "Successful use of Trastuzumab following cardiac resynchronisation therapy," International Journal of Cardiology, vol. 166, no. 2, pp. e33-e34, 2013.

[10] V. L. Roger, "Heart disease, and stroke statistics-2012 update: a report from the American heart association," Japanese Circulation, vol. 125, no. 1, pp. e2-e220, 2012.

[11] T. Callender, M. Woodward, G. Roth et al., "Heart failure care in low- and middle-income countries: a systematic review and metaanalysis," PLoS Medicine, vol. 11, no. 8, Article ID e1001699, 2014.

[12] P. A. Heidenreich, J. G. Trogdon, O. A. Khavjou et al., "Forecasting the future of cardiovascular disease in the United States," Circulation, vol. 123, no. 8, pp. 933-944, 2011.

[13] E. J. Benjamin, "Heart disease and stroke statistics-2017 update: a report from the American heart association," Journal Circulation, vol. 135, no. 10, pp. e146-e603, 2017.

[14] M. D. Ritchey, "Million Hearts: description of the national surveillance and modeling methodology used to monitor the number of cardiovascular events prevented during 2012-2016," Journal of the American Heart Association, vol. 6, no. 5, Article ID e006021, 2017.

[15] G. S. Bloomfield, F. A. Barasa, J. A. Doll, and E. J. Velazquez, "Heart failure in sub-Saharan Africa," Current Cardiology Reviews, vol. 9, no. 2, pp. 157-173, 2013.

[16] Y. G. Tefera, T. M. Abegaz, T. B. Abebe, and A. B. Mekuria, "The changing trend of cardiovascular disease and its clinical characteristics in Ethiopia: hospital-based observational study," Vascular Health and Risk Management, vol. 13, pp. 143-151, 2017.

[17] H. Dokainish, "INTER-CHF Investigators. Global mortality variations in patients with heart failure: results from the international congestive Heart Failure (INTER-CHF) prospective cohort study," Lancet Glob Health, vol. 5, no. 7, pp. e665-72, 2017.

[18] M. U. Sani, G. Cotter, B. A. Davison et al., "Symptoms and signs of heart failure at admission and discharge and outcomes in the sub-saharan acute heart failure (THESUS-HF) registry," Journal of Cardiac Failure, vol. 23, no. 10, pp. 739-742, 2017.

[19] S. Kraus, G Ogunbanjo, K Sliwa, and N. A Ntusi, "Heart failure in sub-Saharan Africa: a clinical approach," South African medical journal = Suid-Afrikaanse tydskrif vir geneeskunde, vol. 106, no. 1, pp. 23-31, 2016.

[20] H. Afnan-Holmes, M. Magoma, T. John et al., "Tanzania's countdown to 2015: an analysis of two decades of progress and gaps for reproductive, maternal, newborn, and child health, to inform priorities for post-2015," The Lancet Global Health, vol. 3, no. 7, pp. e396-e409, 2015. 
[21] A. S. Go, "Executive summary: heart disease and stroke statistics-2013 update: a report from the American Heart Association," Japanese Circulation, vol. 127, no. 1, pp. 143152, 2013.

[22] M. J. Sweeting and S. G. Thompson, "Joint modelling of longitudinal and time-to-event data with application to predicting abdominal aortic aneurysm growth and rupture," Biometrical Journal, vol. 53, no. 5, pp. 750-763, 2011.

[23] H. J. Lim, P. Mondal, and S. Skinner, "Joint modeling of longitudinal and event time data: application to HIV study," Journal of Medical Statistics and Informatics, vol. 1, no. 1, p. 1, 2013.

[24] Q. Chen, R. C. May, J. G. Ibrahim, H. Chu, and S. R. Cole, "Joint modeling of longitudinal and survival data with missing and left-censored time-varying covariates," Statistics in Medicine, vol. 33, no. 26, pp. 4560-4576, 2014.

[25] E. R. Brown, J. G. Ibrahim, and V. DeGruttola, "A flexible Bspline model for multiple longitudinal biomarkers and survival," Journal of Biometrics, vol. 61, no. 1, pp. 64-73, 2005.

[26] L. Wu, L. Wei, Y. Y. Grace, and Y. Huang, "Analysis of longitudinal and survival data: joint modeling, inference methods, and issues," Journal of Probability and Statistics, vol. 2012, Article ID 640153, 17 pages, 2012.

[27] J. Han, E. H. Slate, and E. A. Peña, "Parametric latent class joint model for a longitudinal biomarker and recurrent events," Statistics in Medicine, vol. 26, no. 29, pp. 5285-5302, 2007.

[28] S. Li, "Joint modeling of recurrent event processes and intermittently observed time-varying binary covariate processes," Lifetime Data Analysis, vol. 22, no. 1, pp. 145-160, 2016.

[29] C. Proust-Lima, M. Séne, J. MG Taylor, and H. JacqminGadda, "Joint latent class models for longitudinal and timeto-event data: a review," Statistical Methods in Medical Research, vol. 23, no. 1, pp. 74-90, 2014.

[30] C. Brombin, C. Di Serio, and P. M. Rancoita, "Joint modeling of HIV data in multicenter observational studies: a comparison among different approaches," Statistical Methods in Medical Research, vol. 25, no. 6, pp. 2472-2487, 2016.

[31] M. Sudell, R. Kolamunnage-Dona, and C. Tudur-Smith, "Joint models for longitudinal and time-to-event data: a review of reporting quality with a view to meta-analysis," BMC Medical Research Methodology, vol. 16, no. 1, pp. 168-211, 2016.

[32] J. G. Ibrahim, H. Chu, and L. M. Chen, "Basic concepts and methods for joint models of longitudinal and survival data," Journal of Clinical Oncology, vol. 28, no. 16, pp. 2796-2801, 2010.

[33] L. M. Chen, J. G. Ibrahim, and H. Chu, "Sample size and power determination in joint modeling of longitudinal and survival data," Statistics in Medicine, vol. 30, no. 18, pp. 2295-2309, 2011.

[34] Y. H. Fissuh and G. Muletav, "A joint model for a longitudinal pulse rate and respiratory rate of congestive heart failure patients: at Ayder Referral Hospital of Mekelle University, Tigray, Ethiopia," Journal of Biometrics \& Biostatistics, vol. 6, no. 5, p. 1, 2015.

[35] H. Beck, S. I. Titze, S. Hübner et al., "Heart failure in a cohort of patients with chronic kidney disease: the GCKD study," PLoS One, vol. 10, no. 4, Article ID e0122552, 2015.

[36] T. Nesbitt, S. Doctorvaladan, J. A. Southard et al., "Correlates of quality of life in rural patients with heart failure," Circulation: Heart Failure, vol. 7, no. 6, pp. 882-887, 2014.

[37] O. A. Abdela, A. S Bhagavathula, H Getachew, and Y Kelifa, "Risk factors for developing drug-related problems in patients with cardiovascular diseases attending Gondar University Hospital, Ethiopia," Journal of Pharmacy \& Bioallied Sciences, vol. 8, no. 4, pp. 289-295, 2016.

[38] T. Ahmad, A. Munir, S. H. Bhatti, M. Aftab, and M. A. Raza, "Survival analysis of heart failure patients: a case study," PLoS One, vol. 12, no. 7, Article ID e0181001, 2017.

[39] M. A. Zeru, "Assessment of major causes of heart failure and its pharmacologic management among patients at Felege Hiwot referral hospital in Bahir Dar, Ethiopia," Journal of Public Health and Epidemiology, vol. 10, no. 9, pp. 326-331, 2018.

[40] S. Barlera, L. Tavazzi, M. G. Franzosi et al., "Predictors of mortality in 6975 patients with chronic heart failure in the gruppo italiano per lo studio della Streptochinasi nell'Infarto miocardico-heart failure trial," Circulation: Heart Failure, vol. 6, no. 1, pp. 31-39, 2013.

[41] T. B. Abebe, "Anemia in severe heart failure patients: does it predict prognosis?” BMC Cardiovascular Disorders, vol. 17, no. 1, pp. 1-7, 2017.

[42] A. Hailay, E. Kebede, and K. Mohammed, "Survival during treatment period of patients with severe heart failure admitted to intensive care unit (ICU) at gondar university hospital (GUH), gondar, Ethiopia," American Journal of Health Research, vol. 3, no. 5, pp. 257-269, 2015. 